\title{
A Japanese health success story: trends in cardiovascular diseases, their risk factors, and the contribution of public health and personalized approaches
}

\author{
Hiroyasu Iso
}

Received: 18 January 2011 / Accepted: 14 February 2011 /Published online: 24 March 2011

(C) European Association for Predictive, Preventive and Personalised Medicine 2011

\begin{abstract}
There has been a substantial decline in mortality from stroke and ischemic heart disease (IHD) in Japan between the 1960s and 2000s, which contributed to placing Japanese at the top of world's life expectancy rankings. That mortality decline was attributable to reductions of blood pressure and smoking, in spite of increases in dyslipidemia and diabetes mellitus. The combination of public health and personalized treatment activities to enhance sodium reduction, balanced diets, smoking prevention/cessation as well as hypertension detection and treatment have contributed to the reduction of cardiovascular diseases, demonstrated by a community-based stroke prevention program. The health success story, however, contains an underlying concern about future health threats, the plateaued IHD mortality decline and increased IHD incidence among urban middle-aged men, probably due to increased dyslipidemia and diabetes. The IHD incidence and mortality trends need to be monitored because of a potential future problem for predictive, preventive and personalized medicine.
\end{abstract}

Keywords Stroke - Ischemic heart disease - Risk factors · Trends $\cdot$ Epidemiology $\cdot$ Japan

\section{Introduction}

Japan has achieved the top-rank for longevity in the world; in 2010, the average life expectancy is 79.6 years for men and

H. Iso is National Representative of EPMA in Japan.

H. Iso $(\bowtie)$

Department of Social and Environmental Medicine,

Osaka University Graduate School of Medicine,

2-2 Yamadagaoka,

Suita-shi, Osaka 565-0871, Japan

e-mail: iso@pbhel.med.osaka-u.ac.jp
86.4 years for women. The Japanese longevity is primarily due to the control of acute infectious diseases, malnutrition and tuberculosis between the 1940s and 1960s, and the control of cardiovascular diseases since the 1960s [1].

This health success story in Japan is unique in terms of the reduction of cardiovascular diseases, both of stroke and ischemic heart disease. Other developed countries such as European countries and the United States still have an IHD epidemic with the high prevalence of obesity, diabetes mellitus, and dyslipidemia (high triglycerides and low HDL-cholesterol) [2]. On the other hand, many developing countries, while in the process of conquering malnutrition and infectious disease, have encountered emerging cardiovascular diseases along with rapidly increasing prevalence of obesity, diabetes, hypertension, and dyslipidemia [3].

From the viewpoint of predictive, preventive and personalized medicine, the Japanese health success story in the prevention of cardiovascular diseases may be of value for many developed and developing countries in building effective population and individual strategies for the prevention and control of cardiovascular diseases. To this end, we reviewed mortality and incidence trends in cardiovascular diseases, risk factors, and related Japanese lifestyles over the past decades (as summarized in Table 1), and the public health and individual personalized approaches for prevention and control of cardiovascular diseases.

\section{Trends in mortality from stroke and ischemic heart disease}

After being ranked the top cause of death between 1951 and 1980, stroke is now the third-ranked cause of death in Japan, following cancer and heart disease [1, 2]. Age-adjusted mortality from stroke declined by $80 \%$ for men and $87 \%$ for women aged 55-79 years between 1950 and 1997, with a 
Table 1 Trends in cardiovascular diseases and their risk factors in Japan between the 1960s and 2000s

\begin{tabular}{lll}
\hline & $1990 \mathrm{~s}$ & $2000 \mathrm{~s}$ \\
\hline $\begin{array}{l}\text { Cardiovascular disease mortality } \\
\text { Stroke }\end{array}$ & High & $\begin{array}{l}\text { Moderate } \\
\text { Low }\end{array}$ \\
IHD & Low & \\
Cardiovascular disease incidence & & Moderate \\
Stroke & High & Low \\
IHD & Low & \\
Risk factors & & Moderate \\
Blood pressure & High & Moderate \\
Smoking, men & High & Low \\
Smoking, women & Low & Moderate \\
Total cholesterol & Low & Moderate \\
Diabetes mellitus, men & Low & Low \\
Diabetes mellitus, women & Low & Moderate \\
Overweight, men & Low & Low \\
Overweight, women & Low & Moderate \\
Alcohol, men & High & Low \\
Alcohol, women & Low & \\
\hline
\end{tabular}

${ }^{a}$ With increasing trend in urban middle-aged men

large decline between the 1960s and 1980s [3], the decline has slowed down thereafter [1-3]. According to the WHO database, the age-adjusted annual stroke mortality in 2004 was 52 per 100,000 for men and 34 per 100,000 for women of all ages [2].

Mortality from IHD comprises approximately half of mortality from heart disease. Age-adjusted mortality from IHD declined $50 \%$ for men and $65 \%$ for women between 1969 and 1992 [3], and has continued to decline [1, 2]. According to the WHO database, the age-adjusted annual IHD mortality rate in 2004 was 45 per 100,000 for men and 21 per 100,000 for women [2]. Japan's IHD mortality was the lowest among developed countries, and was $1 / 3$ to $1 / 5$ of that of the United States and the United Kingdom [2].

The mortality decline for IHD was smaller among men residing in the Tokyo and Osaka metropolitan areas than among those in the rest of Japan [4]. Men aged 30-49 years in the two largest metropolitan cities of Tokyo and Osaka had no substantial change in mortality (approximately 10 per 100,000; declined slightly between the 1960s and the 1980s and plateaued thereafter) while those in the rest of Japan showed a steady decline.

\section{Trends in incidence of stroke and ischemic heart disease}

Several population-based studies showed long-term trends in the incidence of stroke between the 1960 and the 2000s $[5,6]$. The incidence of stroke was very high among Akita rural residents aged 40-69 years in 1964-1971 (9.7 per 1,000 person-years for men and 4.2 per 1,000 person-years for women) and had declined by about $75 \%$ by $1996-2003$ (2.3 per 1,000 person-years for men and 1.1 per 1,000 person-years for women) [5]. Among Osaka urban residents aged 40-69 years, the incidence of stroke was moderate in 1964-1971 (2.7 per 1,000 person-years for men and 1.3 per 1,000 person-years for women) and had declined by $56 \%$ and 36\% respectively, by 1996-2003 (1.2 per 1,000 personyears for men and 0.8 per 1,000 person-years for women) [5]. In the town of Hisayama in Kyushu, another rural community, the incidence of stroke declined by $56 \%$ for men and $35 \%$ for women from the 1961 cohort (1.2 per 1,000 person-years for men and 0.5 per 1,000 person-years for women) to the 1988 cohort ( 0.6 per 1,000 person-years for men and 0.4 per 1,000 person-years for women) [6].

The incidence of IHD has generally been low in most Japanese populations: 1 to 2 per 1,000 person-years for men and 0.1 to 1 per 1,000 person-years for women aged 40-69 years, with two- to five- times higher incidence for men than for women [5-8]. The IHD incidence has not changed substantially in Japanese populations in rural communities nor in the Hiroshima/Nagasaki cohort during the past three decades [5-7]. However, there is emerging evidence of an incidence increase among urban male populations. Male workers aged 40-59 years in Osaka, one of the top 3 metropolitan cities in Japan, showed an increasing IHD incidence from 0.4 per 1,000 person-years in 1963-1970 to 1.5 per 1,000 person-years in 1979-1986, and then plateaued during 1987-1994 [9]. Male residents aged 40-69 years in Osaka had an increasing IHD incidence from 0.6 per 1,000 person-years in 1980-1987 to 1.3 per 1,000 person-years in 1996-2003 [5]. Male residents of all ages in Takashima City, a medium-size city, had an increasing IHD incidence from 0.7 per 1,000 person-years in 1990-1992 to 1.0 per 1,000 person-years in 1999-2001 [10].

\section{Vascular pathology in Japanese and Western populations}

The remarkable difference in cardiovascular profiles between Japan and the North American and European countries may be attributable to different proportions of two major vascular pathologies occurring in small and large arteries. The small artery pathology is referred to as arteriosclerosis (or arteriolosclerosis), typically observed in penetrating arterioles in basal ganglions of the brain. This pathology is characterized by necrosis or apoptosis of smooth muscle cells within the media, leading to the formation of micro-aneurysms (eventually intraparenchymal hemorrhage) and fibrous proliferative changes (eventually lacunar stroke) (right side of Fig. 1). The large artery pathology is referred to as atherosclerosis, 
Fig. 1 Vascular pathology and risk factors for small and large arteries: arteriosclerosis (right) and atherosclerosis (left) [12]

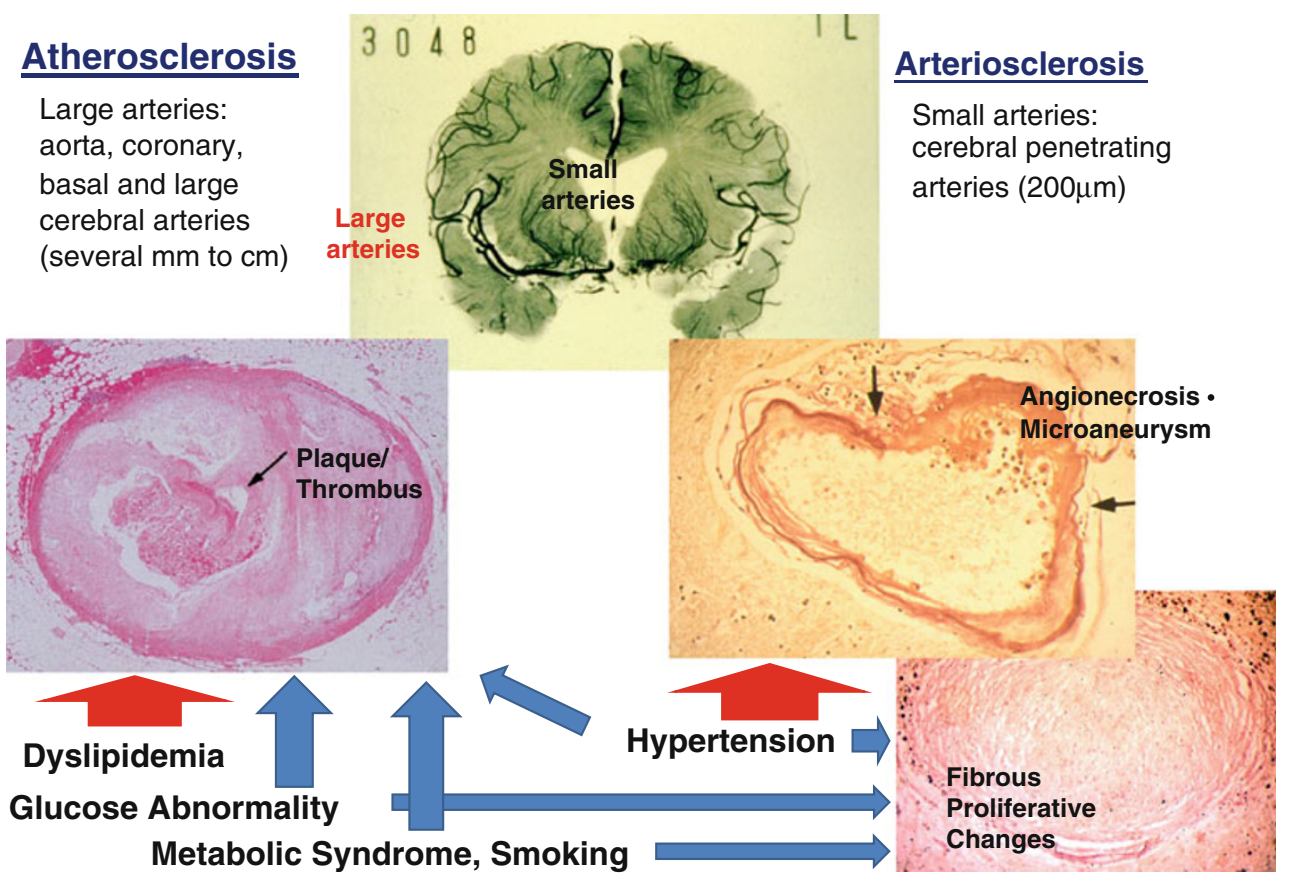

typically observed in aorta, coronary arteries, carotid arteries, basal cerebral arteries and large cerebral arteries. This pathology is characterized by lipid accumulation with proliferative changes leading to plaque formation (eventually IHD) (left side of Fig. 1) [11, 12]. The major risk factor for arteriosclerosis is primarily hypertension, while those for atherosclerosis are dyslipidemia, glucose abnormality, and smoking, as well as hypertension and metabolic syndrome.

Arteriosclerosis has been dominant in Japanese populations, reflecting high stroke mortality, while the same is true for atherosclerosis, reflecting high IHD mortality in European and US populations [12]. The recent rise in IHD incidence among urban middle-aged men, however, suggests a potential increase in atherosclerosis among Japanese subpopulations [12]. Further, the proportions of stroke subtypes also vary between Japanese and European/ US populations. The proportions of stroke subtypes for Japanese populations were approximately 20-30\% for intraparenchymal hemorrhage, $40 \%$ for lacunar infarction (both of which are based primarily on arteriosclerosis) and $20 \%$ for large-artery occlusive infarction (based primarily on atherosclerosis), while those for Western populations were $10-20 \%, 20 \%$ and $50 \%$, respectively [13].

These national differences in vascular pathology and cardiovascular diseases, although genetic contributions may exist, are probably due to major differences in lifestyles and, in particular, diet. High sodium, low calcium, low animal protein intakes, and, for middle-aged men, high alcohol consumption, may have contributed to the high prevalence of hypertension and high mortality from stroke among Japanese populations [14-19]. On the other hand, low saturated fat (meat) and high n-3 polyunsaturated fat (fish) intakes have contributed to the low prevalence of hypercholesterolemia and low mortality from IHD among Japanese populations [20-22].

\section{Trends in risk factors for stroke and ischemic heart disease, and their related lifestyles}

High blood pressure

High blood pressure, in particular high systolic blood pressure, is the strongest risk factor for stroke and each stroke subtype [23] and one of the major risk factors for IHD [24]. According to Japanese national surveys, mean systolic blood pressure for persons aged $\geq 30$ years declined substantially from $142 \mathrm{mmHg}$ in 1961 to $135 \mathrm{mmHg}$ in 2007 for men, and from $141 \mathrm{mmHg}$ to $126 \mathrm{mmHg}$ for women, while changes in mean diastolic blood pressure levels were not large: from $82 \mathrm{mmHg}$ to $83 \mathrm{mmHg}$ for men, and from $81 \mathrm{mmHg}$ to $77 \mathrm{mmHg}$ for women [25, 26]. However, there is still a high prevalence of high blood pressure (systolic blood pressure $\geq 140 \mathrm{mmHg}$ and/or diastolic blood pressure $\geq 90 \mathrm{mmHg}$ ), which, in 2000, was $52 \%$ for men and $40 \%$ for women aged $\geq 30$ years [26]. Similar blood pressure trends were observed in several population-based studies where the prevalence of antihypertensive medication use rose substantially from $4-7 \%$ in the $1960 \mathrm{~s}$, to $13-26 \%$ in the $1970 \mathrm{~s}$, but did not change substantially thereafter [5]. This suggests the major contribution lifestyle changes, such as a decrease in sodium intake, an increase in calcium and animal protein intakes 
[25], made to the reduction of blood pressure levels [1418]. Miura K. discusses the importance of high blood pressure as a risk factor for cardiovascular diseases, their trends, and related factors in detail in the present issue of the EPMA journal [27].

\section{Smoking}

Smoking is a risk factor for some of the stroke subtypes, such as subarachnoid hemorrhage, large-artery occlusive infarction, and to a lesser extent lacunar stroke, but not intraparenchymal hemorrhage [28], while smoking is a strong risk factor for IHD [29]. Smoking cessation benefits include risk reduction of cardiovascular diseases within several years [30].

According to Japanese national surveys, the prevalence of active smoking has declined from $82 \%$ in 1965 , to $46 \%$ in 2005 for men aged $\geq 20$ years, while that for women declined slightly from $16 \%$ in 1965 , to $12 \%$ in 2005 , with an increase from $7 \%$ to $21 \%$ for ages $20-29$ years [31]. More recently, there has been a steeper decline in the smoking prevalence for men, but not for women (from $46 \%$ in 2005 to $39 \%$ in 2007 for men, and from $12 \%$ to $11 \%$ for women) partly due to the Health Promotion Act, which banned smoking in public places [1].

\section{Blood lipids}

High serum total cholesterol has not been associated with risk of stroke, but is an established risk factor for IHD [32, 33]. Low serum HDL-cholesterol is also an established risk factor for IHD and a risk factor for ischemic stroke, probably both of lacunar and large-artery occlusive infarction [34]. High serum triglyceride is also a risk factor for IHD [34] and ischemic stroke [35].

According to Japanese national surveys, mean total serum cholesterol levels increased from $186 \mathrm{mg} / \mathrm{dl}$ in 1980 , to $199 \mathrm{mg} / \mathrm{dl}$ in 1990 , and $200 \mathrm{mg} / \mathrm{dl}$ in 2000 among men aged $\geq 30$ years, and from $191 \mathrm{mg} / \mathrm{dl}$, to $207 \mathrm{mg} / \mathrm{dl}$, and $208 \mathrm{mg} / \mathrm{dl}$ among women of the same age [25]. The increase in mean total cholesterol plateaued thereafter at $199 \mathrm{mg} / \mathrm{dl}$ for men and $208 \mathrm{mg} / \mathrm{dl}$ for women in 2007 [25]. Mean serum HDL-cholesterol levels have been higher for people of Japanese ethnicity than for those of Caucasian decent [36] and increased from $50 \mathrm{mg} / \mathrm{dl}$ in 1990, to $53 \mathrm{mg} / \mathrm{dl}$ in 2000 , and $58 \mathrm{mg} / \mathrm{dl}$ in 2007 for men, and from $53 \mathrm{mg} / \mathrm{dl}$, to $61 \mathrm{mg} / \mathrm{dl}$, and $68 \mathrm{mg} / \mathrm{dl}$ for women $[25,26]$. Also, mean serum triglyceride levels increased between 1990 and 2000 for both men and women [26]. Several populationbased studies indicated that the prevalence of the use of lipid-lowering medication increased between the 1980s and the 2000 s by up to $3 \%$ for men and $9 \%$ for women $[5,8]$. Kokubo Y. discusses the importance of dyslipidemia as a risk factor for IHD and ischemic stroke in more detail in the present issue of the EPMA journal [37].

Diabetes mellitus

Type 2 diabetes mellitus is an established risk factor for IHD and a risk factor for ischemic stroke, probably both of large artery occlusive infarction and lacunar infarction [38, 39]. The prevalence of type 2 diabetes $(\mathrm{HbA} 1 \mathrm{c} \geq 6.1 \%$ and/or medication use) increased slightly for men aged $\geq 20$ years (10\% in $1997,13 \%$ in 2002 , and $15 \%$ in 2007 ), but did not change for women ( $7 \%$ in the three periods) $[25,38]$. According to community-based studies, the prevalence of diabetes increased from $2-8 \%$ in the 1980 s to $6-13 \%$ in the 1990 s among middle-aged men, and from $1-5 \%$ to $3-9 \%$ among middle-aged women [40, 41]. Kokubo Y. discusses the importance of impaired fasting glucose as well as diabetes as a risk factor for IHD and ischemic stroke in detail in the present issue of the EPMA journal [37].

\section{Being overweight and physical inactivity}

Being overweight is a risk factor for IHD and ischemic stroke, and probably large artery occlusive infarction, but the independency of other risk factors, such as hypertension, dyslipidemia, and glucose abnormality, is uncertain [42]. However, being overweight leads to the development of these risk factors and obstructive sleep apnea [43] which are regarded as important targets in the prevention of cardiovascular diseases. Metabolic syndrome, abdominal obesity with a constellation of high blood pressure, dyslipidemia and glucose abnormality have been recognized as risk factors for IHD and ischemic stroke [35]. Physical inactivity has been associated with increased risk of IHD [44]. Tanigawa T. presents an overview of the impact of obstructive sleep apnea on diabetes, hypertension, metabolic syndrome, and cardiovascular diseases in the present issue of the EPMA journal [45].

Mean BMI and the prevalence of being overweight have increased consistently among men since the 1980 s, but did not change among women according to Japanese national surveys $[25,26]$. Mean BMI stayed at $22.5 \mathrm{~kg} / \mathrm{m}^{2}$ in 1980 , and $22.5 \mathrm{~kg} / \mathrm{m}^{2}$ in 1990 , increasing to $23.4 \mathrm{~kg} / \mathrm{m}^{2}$ in 2000 , and $23.7 \mathrm{~kg} / \mathrm{m}^{2}$ in 2007 and the prevalence of being overweight (BMI $\geq 25.0 \mathrm{~kg} / \mathrm{m}^{2}$ ) increased from $19 \%$, to $23 \%, 28 \%$, and $31 \%$ for men $\geq 30$ years old, and the corresponding mean values and prevalence for women were from $22.8 \mathrm{~kg} / \mathrm{m}^{2}, 22.8 \mathrm{~kg} / \mathrm{m}^{2}, 22.8 \mathrm{~kg} / \mathrm{m}^{2}$, and $22.5 \mathrm{~kg} / \mathrm{m}^{2}$, and $23 \%, 23 \%, 24 \%$, and $22 \%$, respectively.

Although no data is available on long-term trends in physical activity among Japanese populations, a decline in physical activity is suggested, in particular for men, because of stable or decreased energy intakes [46] and increased BMI accompanying mechanization of the work 
environment and motorization [47]. In the present issue of the EPMA journal, Yatsuya $H$. et al. discusses the impact of being overweight on high blood pressure, the risks of IHD, ischemic stroke and its subtypes in detail [48].

\section{Alcohol intake}

Light to moderate alcohol intake has been associated with a $30-60 \%$ reduced risk of IHD and ischemic stroke compared with non-drinkers [49-51].

No Japanese national surveys have examined long-term trends in alcohol intake, but the prevalence of heavy drinkers ( $\geq 46$ g ethanol per day) was $22 \%$ for men of ages $\geq 20$ years and $6 \%$ for women of the same age in 2007 [25]. A population-based study showed that the prevalence of heavy drinkers declined, while the prevalence of light-to-moderate drinkers ( 1 to $45 \mathrm{~g}$ ethanol per day) increased between the 1980s and the 1990s among both rural and urban men [52].

\section{Community-based prevention program for cardiovascular diseases: the evidence on the health effect by the combination of those public health and personalized activities}

Because of the high prevalence of hypertension and high mortality from stroke in Japanese populations, the prevention of hypertension and control in communities have been justified for the prevention of stroke [53]. We evaluated a community-based prevention program in the full intervention community, between 1964 and 1987, with reference to a control community (the minimum intervention community) with a similar distribution of age, sex, job, and geographical area [54]. The program was the combination of population and individual approaches for the prevention and control of hypertension. Hypertension prevention was attempted to be done by population-wide health education campaign through cable radio, newsletter, pamphlet, billboard, and health professionals and volunteers. Hypertension control was performed by free systematic blood pressure screening, referral to local physicians, and lifestyle improvements with the support of local government and health volunteers. Local physicians performed every effort to make personalized treatment for hypertensive patients by their advice on lifestyle modification and prescription of antihypertensive medication.

The health effect by the combination of those public health and personalized activities appeared in terms of a high maintaining screening rate and larger decline in stroke incidence in the full intervention community than in the minimum intervention community. The full intervention community maintained a high health screening rate $(70-80 \%$ in men and $80-90 \%$ in women) with satisfactory referral, health education and greater blood pressure control, while the control community had a declining health screening rate (from $80 \%$ to $50 \%$ in men and from $90 \%$ to $60 \%$ in women). Stroke incidence declined by a larger amount for men in the full intervention community ( $75 \%$ decline) than for men in the control group ( $29 \%$ decline) over time, whereas stroke incidence declined by $45 \%$ for women in both communities (Fig. 2). The lack of differential changes in stroke incidence in women may be due to their elevated levels of health consciousness and adherence to the health program even if the intervention was minimal. Delivery of a hypertension control service through intensive community-based screening supplemented by health education and citizen support appeared to be effective in the prevention of stroke.

\section{National program for prevention of cardiovascular diseases: the health policy reforms in Japan}

The national prevention program for cardiovascular diseases was launched in 1982 under the Health Service for the Elderly Act based on the establishment of national health
Fig. 2 Sex-specific and age-adjusted stroke incidence in the full intervention community and the minimum intervention community, men and women aged $\geq 30$ years. Difference from the minimum intervention community: $\dagger P<0.01$, $\$ P<0.001[54]$

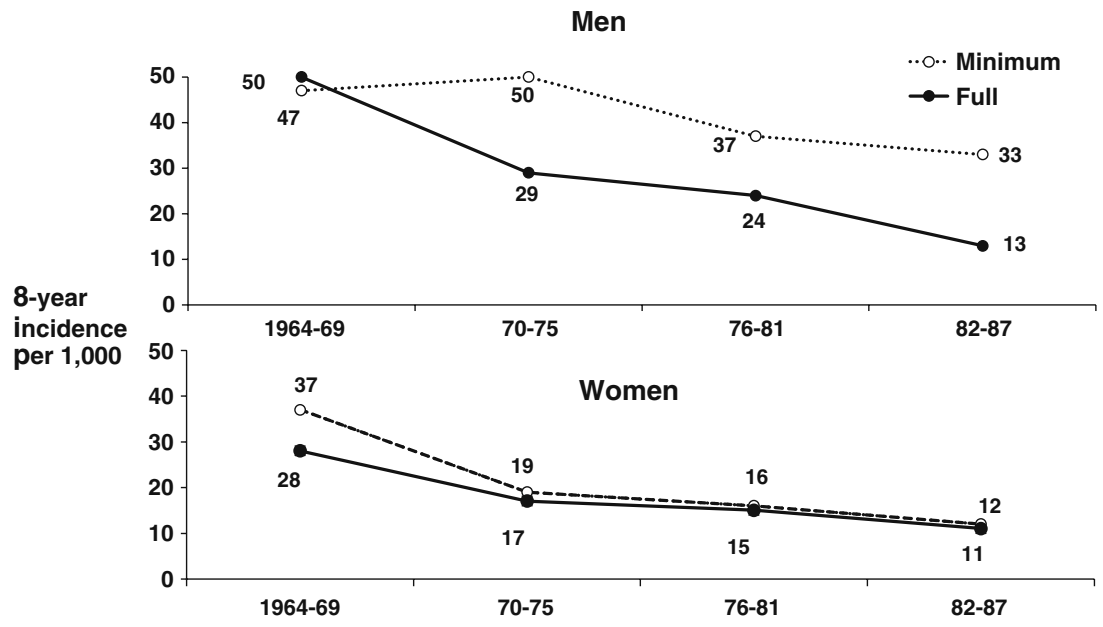


insurance coverage since 1961 [1]. Under this Act, insurers are municipal governments, and are financed with help from prefectural and national governments to conduct health screening and health education for residents aged 40 and over, as well as care for the aged. The Industrial Safety and Health Act since 1972 has assured health screening and education for company employees. The universal insurance coverage and health screening and education system enhanced the hypertension control and lifestyle modification for highrisk individuals in company and community settings.

Meanwhile, the national government performed a series of revisions to the Medical Care Act in 1985, 1992, 1997, and 2000 to control the number of beds, to differentiate hospital roles as high-technology hospitals, long-term care hospitals, and regional tertiary hospitals, to emphasize the importance of informed consent and staff training, and to enhance amenities. That act has improved the operation of local ambulance system that allowed patients with acute cardiovascular events such as stroke and heart attack admitted quickly to local acute care hospitals.

Because of rapid aging and high medical costs for the elderly, the national government amended the Long-term Care Insurance Act in 1997 and the Health Promotion Act in 2002 to improve people's lifestyles in line with the goals set out in Healthy People Japan 2000.

In 2006, the Japanese government amended a reform act regarding the health care system for the prevention and treatment of lifestyle-related diseases to slow down the rise in medical expenditure. The Health Service for the Elderly Act since 1982 has evolved into the Elderly Health Care Security Act, where the health insurer is a central health care provider body rather than municipal governments. Under this
Act, the emphasis is placed on the prevention and control of metabolic syndrome where abdominal obesity is an essential component within a constellation of high blood pressure, dyslipidemia, and glucose abnormality. Although persons with metabolic syndrome are at high risk of ischemic stroke and IHD, non-obese persons with high blood pressure, dyslipidemia, or glucose abnormality have a similar or higher prevalence, and are also at high risk.

Figure 3 illustrates the results from recent large cohort studies on the relationship of metabolic syndrome with the incidence of and mortality from cardiovascular disease [55, 56]. Abdominal obesity was substituted with body mass index $\geq 25 \mathrm{~kg} / \mathrm{m}^{2}$, and the classification of metabolic syndrome based on the Japanese [57] or International Federation of Diabetes [58] criteria corresponded to a body mass index $\geq 25 \mathrm{~kg} / \mathrm{m}^{2}$ plus two metabolic risk factors. The population attributable risk fraction (the percentage of preventable disease by control of target exposure) was similar or even greater in non-obese individuals with two risk factors than those with metabolic syndrome. The pitfalls for the application of the Japanese or International Federation of Diabetes criteria in predictive, preventive, and personalized medicine have also been addressed by other Japanese, Korean, and European studies [59-63], and are discussed by Yatsuya H. et al. in the present issue of the EPMA journal [48].

\section{Factors explaining the unique trends in cardiovascular diseases in Japan}

Japan is unique among developed countries in that it has achieved a substantial reduction of both stroke and IHD
Fig. 3 Sex-specific hazard ratios and populationattributable fractions of cardiovascular diseases according to the number of components of the metabolic syndrome (excluding overweight), stratified by overweight (BMI $\geq 25 \mathrm{~kg} / \mathrm{m}^{2}$ ). Incidence outcomes are from the JPHC Study while mortality outcomes are from the IPH Study $[55,56]$

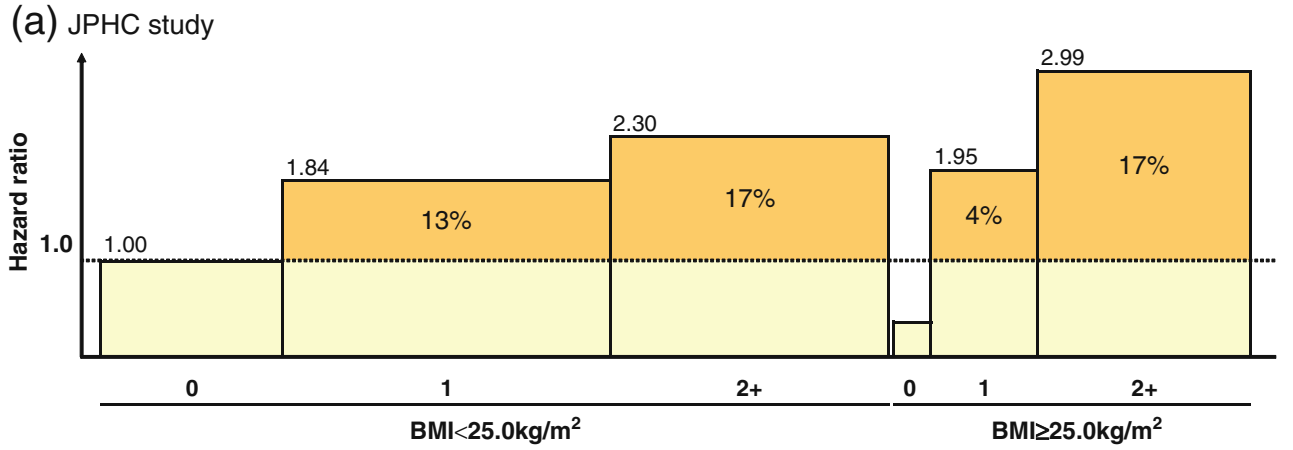

(b) IPH study

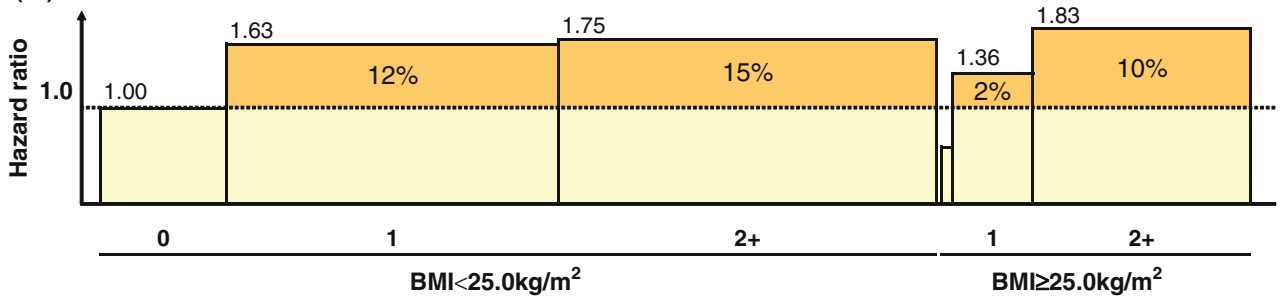


mortality since the 1960s. Meanwhile, there have been remarkable changes in risk factors and related lifestyles. Mean systolic blood pressure levels for men and women, and the prevalence of smoking among men declined, but mean serum total cholesterol and triglycerides increased for men and women. The decline in stroke and IHD mortality has been attributable mostly to the large declines in high blood pressure and smoking. These risk factor reductions may be canceled out by the potentially adverse effects of increases in total cholesterol levels, triglycerides and glucose abnormality over the past decades. Furthermore, high total cholesterol would need a longer incubation period to exert a maximum effect on risk of IHD [64].

The community-based prevention program for stroke has been shown as feasible and demonstrated to be effective for the accelerated reduction of stroke incidence through intensive community-based screening for hypertension, supplemented by health education and broad citizen support.

The overall low IHD incidence among Japanese men and women, however, can be explained by favorable lipid profiles and glucose metabolism along with lower BMI levels compared with Western populations. Lifestyle factors including low total and saturated fat intakes $[5,8,65]$, high fish intake $[21,22]$ and high soy intake $[46,66]$ for men and women, and light-to-moderate alcohol intake [49-52] for men, may be major contributing factors.

The trends in IHD mortality did vary across gender, middle and older ages, and rural and urban populations, while stroke morality declined consistently. In the largest metropolitan cities of Tokyo and Osaka, where $17 \%$ of the total national population reside, the IHD mortality decline was smaller for younger middle-aged men compared to that of those residing in the rest of Japan. The IHD incidence tripled among urban male workers and doubled for urban male residents, and increased by $50 \%$ for semi-urban male residents. The higher sustained cholesterol levels plus a recent rise in diastolic blood pressure along with declines in systolic blood pressure and the prevalence of smoking may explain in part the slowed-down decline in IHD mortality among middle-aged urban men, and increased IHD incidence among urban men. Urban men are reported to have a higher fat intake than rural men (22\% to $26 \%$ versus $19 \%$ of total energy for ages $40-59$ years) $[5,8]$ as well as a lower level of physical activity ( 1,600 versus 1,800 calories expended per day) [67].

The IHD mortality decline in conjunction with an increase or no change in IHD incidence in Japan may be accounted for by improvements in medical treatment and/or decreased severity of IHD during the past decades. The number of emergency medical centers equipped with an ICU or CCU in Japan stood at 17 in 1978, 103 in 1989, and 201 in 2007 [1]. The in-hospital case-fatality rates at CCU hospitals declined by over $50 \%$ between the early 1980 s and the late $1990 \mathrm{~s}$, probably due to improved medical treatment [68]. A series of autopsy studies showed a decline in the coronary atherosclerosis score for Japanese men and women between the 1960s and the 1980s, suggesting a decline in IHD severity [69].

\section{Outlook}

The threat of increasing incidence of IHD among urban men may justify the recent amendment of the Elderly Health Care Security Act, focusing on preventive and control activities targeting metabolic syndrome. However, it should be noted that the public health burden of cardiovascular disease was equal or even greater among non-obese high-risk individuals compared with obese high-risk individuals. This finding supports an emphasis on prevention and control programs for non-obese high-risk individuals as well as obese high-risk individuals to achieve communitywide prevention and control of cardiovascular diseases. The activities under the Elderly Health Care Security Act focusing on metabolic syndrome need to be re-evaluated and modified in the near future.

\section{Concluding remarks}

There have been distinctive and overall favorable trends in mortality, incidence, and risk factors for stroke and IHD in Japan, indicating a Japanese health success story in the areas of cardiovascular diseases and increasing longevity. However, middle-aged men, especially in urban areas, could be the victims of an impending IHD epidemic, as is the case in some developing countries [70]. The potential epidemic needs to be monitored by continued surveillance, and points to an important issue in predictive, preventive, and personalized medicine.

\section{References}

1. Japan Health and Welfare Statistics Association. Kokumin-eisei no Douko (Trends for National Hygiene) 2008. J Health and Welfare Stat 2008 (in Japanese).

2. WHO Collaborating Centre for Surveillance of Cardiovascular Disease: Global Cardiovascular Infobase. http://www.cvdinfobase. ca/. Accessed Jan 1, 2011.

3. Liu L, Ikeda K, Yamori Y. Changes in stroke mortality rates for 1950 to 1997. A great slowdown of decline trend in Japan. Stroke. 2001;32:1745-9.

4. Okayama A, Ueshima H, Marmot M, Elliott P, Choudhury SR, Kita Y. Generational and regional differences in trends of mortality from ischemic heart disease in Japan from 1969 to 1992. Am J Epidemiol. 2001;153:1191-8. 
5. Kitamura A, Sato S, Kiyama M, Imano H, Iso H, Okada T, et al. Trends in the incidence of coronary heart disease and stroke and their risk factors in Japan, 1964 to 2003: the Akita-Osaka Study. J Am Coll Cardiol. 2008;52:71-9.

6. Kubo M, Kiyohara Y, Kato I, Tanizaki Y, Arima H, Tanaka K, et al. Trends in the incidence, mortality, and survival rate of cardiovascular disease in a Japanese community: the Hisayama study. Stroke. 2003;34:2349-54.

7. Kodama K, Sasaki H, Shimizu Y. Trend of coronary heart disease and its relationship to risk factors in a Japanese population: a 26year follow-up, Hiroshima/Nagasaki study. Jpn Circ J. 1990;54:414-21.

8. Ishikawa S, Kayaba K, Gotoh T, Nago N, Nakamura Y, Tsutsumi A, et al. Incidence of total stroke, stroke subtypes, and myocardial infarction in the Japanese population: the JMS Cohort Study. J Epidemiol. 2008;18:144-50

9. Kitamura A, Iso H, Iida M, Naito Y, Sato S, Jacobs DR, et al. Trends in the incidence of coronary heart disease and stroke and the prevalence of cardiovascular risk factors among Japanese men from 1963 to 1994. Am J Med. 2002;112:104-9.

10. Rumana N, Kita Y, Turin TC, Murakami Y, Sugihara H, Morita Y, et al. Trend of increase in the incidence of acute myocardial infarction in a Japanese population; Takashima AMI Registry, 1990-2001. Am J Epidemiol. 2008;167:1358-64.

11. Konishi M, Iso H, Komachi Y, Iida M, Shimamoto T, Jacobs Jr DR, et al. Associations of serum total cholesterol, different types of stroke, and stenosis distribution of cerebral arteries. The Akita Pathology Study. Stroke. 1993;24:954-64.

12. Iso H. Lifestyle and cardiovascular disease in Japan. J Atheroscl Thromb 2011;18:83-8.

13. Kitamura A, Nakagawa $Y$, Sato M, Iso H, Sato S, Imano H, et al. Proportions of stroke subtypes among men and women $>$ or $=40$ years of age in an urban Japanese city in 1992, 1997, and 2002. Stroke. 2006;37:1374-8.

14. Umesawa $M$, Iso $H$, Date $C$, Yamamoto $A$, Toyoshima $H$, Watanabe $\mathrm{Y}$, et al. Relations between dietary sodium and potassium intakes and mortality from cardiovascular disease: the Japan Collaborative Cohort Study for Evaluation of Cancer Risks. Am J Clin Nutr. 2008;88:195-202.

15. Takachi R, Inoue M, Shimazu T, Sasazuki S, Ishihara J, Sawada $\mathrm{N}$, et al. Consumption of sodium and salted foods in relation to cancer and cardiovascular disease: the Japan Public Health Center-based Prospective Study. Am J Clin Nutr. 2010;91:456-64.

16. Umesawa $M$, Iso $H$, Date $C$, Yamamoto $A$, Toyoshima $H$, Watanabe $\mathrm{Y}$, et al. Dietary intake of calcium in relation to mortality from cardiovascular disease: the JACC Study. Stroke. 2006;37:20-6.

17. Umesawa M, Iso H, Ishihara J, Saito I, Kokubo Y, Inoue M, et al. Dietary calcium intake and risks of stroke, its subtypes, and coronary heart disease in Japanese: the JPHC Study Cohort I. Stroke. 2008:39:2249-56.

18. Umesawa $M$, Sato $S$, Imano $H$, Kitamura $A$, Shimamoto $T$, Yamagishi K, et al. Relations between protein intake and blood pressure in Japanese men and women: the Circulatory Risk in Communities Study (CIRCS). Am J Clin Nutr. 2009;90:377-84.

19. Iso H, Sato S, Kitamura A, Naito Y, Shimamoto T, Komachi Y. Fat and protein intakes and risk of intraparenchymal hemorrhage among middle-aged Japanese. Am J Epidemiol. 2003;157:32-9.

20. Ueshima H, Iida M, Shimamoto T, Konishi M, Tanigaki M, Doi $\mathrm{M}$, et al. Dietary intake and serum total cholesterol level: their relationship to different lifestyles in several Japanese populations. Circulation. 1982;66:519-26.

21. Iso H, Kobayashi M, Ishihara J, Sasaki S, Okada K, Kita Y, et al. JPHC Study Group. Intake of fish and n 3 fatty acids and risk of coronary heart disease among Japanese: the Japan Public Health Center-Based (JPHC) Study Cohort I. Circulation. 2006;113:195-202.
22. Yamagishi K, Iso H, Date C, Fukui M, Wakai K, Kikuchi S, et al. for the JACC Study Group. Fish, $\omega-3$ polyunsaturated fatty acids, and mortality from cardiovascular diseases in a nationwide community-based cohort of Japanese: the JACC Study. J Am Coll Cardiol. 2008;52:988-96.

23. Asian Pacific Cohort Studies Collaboration. Systolic blood pressure, diabetes and the risk of cardiovascular disease in the Asia-Pacific region. J Hypertens. 2007;25:1205-13.

24. van den Hoogen PC, Feskens EJ, Nagelkerke NJ, Menotti A, Nissinen A, Kromhout D. The relation between blood pressure and mortality due to coronary heart disease among men in different parts of the world. N Engl J Med. 2000;342:1-8.

25. Japan Ministry of Health, Labor and Welfare. The National Health and Nutrition Surveys 2007. Tokyo: Daiichi Shuppan Press; 2010.

26. Japan Ministry of Health, Labor and Welfare. National Survey on Circulatory Disorders in 1980, 1990 and 2000. 1983, 1993 and 2003 (in Japanese, English abstract).

27. Miura K. Epidemiology and prevention of hypertension in Japanese: how could Japan get longevity? EPMA J. 2011. doi:10.1007/s13167-011-0069-y.

28. Mannami T, Iso H, Baba S, Sasaki S, Okada K, Konishi M, et al. Cigarette smoking and risk of stroke and its subtypes among middle-aged Japanese men and women: the JPHC Study Cohort I. Stroke. 2004;35:1248-53.

29. Baba S, Iso H, Mannami T, Sasaki S, Okada K, Konishi M, et al. Cigarette smoking and risk of coronary heart disease incidence among middle-aged Japanese men and women: the JPHC Study Cohort I. Eur J Cardiovasc Prev Rehabil. 2006;13:207-13.

30. Honjo K, Iso H, Tsugane S, Tamakoshi A, Satoh H, Tajima K, et al. The effects of smoking and smoking cessation on mortality from cardiovascular disease among Japanese: pooled analysis of three large-scale cohort studies in Japan. Tob Control. 2010;19:50-7.

31. Japan Ministry of Health, Labor and Welfare. Smoking and Health. Committee Report on Smoking and Health Problems. Tokyo: Hoken Dojin; 2002. in Japanese.

32. Cui R, Iso H, Toyoshima H, Date C, Yamamoto A, Kikuchi S, et al. Serum total cholesterol levels and risk of mortality from stroke and coronary heart disease in Japanese. Atherosclerosis. 2007; 194:415-20.

33. Okamura T, Tanaka H, Miyamatsu N, Hayakawa T, Kadowaki T, Kita $\mathrm{Y}$, et al. The relationship between serum total cholesterol and all-cause or cause-specific mortality in a 17.3-year study of a Japanese cohort. Atherosclerosis. 2007;190:216-23.

34. Iso H, Naito Y, Sato S, Kitamura A, Okamura T, Sankai T, et al. Serum triglycerides and risk of coronary heart disease among Japanese men and women. Am J Epidemiol. 2001;153:490-9.

35. Iso H, Sato S, Kitamura A, Imano H, Kiyama M, Yamagishi K, et al. Metabolic syndrome and the risk of ischemic heart disease and stroke among Japanese men and women. Stroke. 2007;38:174451.

36. Ueshima H, Iida M, Shimamoto $T$, Konishi M, Tanigaki M, Nakanishi N, et al. High-density lipoprotein-cholesterol levels in Japan. JAMA. 1982;247:1985-7.

37. Kokubo Y. Associations of impaired glucose metabolism and dyslipidemia with cardiovascular diseases: what have we learned from Japanese cohort studies for individualized prevention and treatment? EPMA J. 2011. doi:10.1007/s13167-011-0074-1.

38. Japan Ministry of Health, Labor and Welfare. The National Survey for Diabetes Mellitus 2002. http://www..mhlw.go.jp/ toukei/kouhyo/indexkk_4_2.htm. Accessed 1, Jan 2011.

39. Fujishima M, Kiyohara $Y$, Kato I, Ohmura T, Iwamoto $H$, Nakayama K, et al. Diabetes and cardiovascular disease in a prospective population survey in Japan: the Hisayama Study. Diabetes. 1996;45 Suppl 3:S14-6.

40. Ohmura T, Ueda K, Kiyahara Y, Kato I, Iwamoto H, Nakayama $\mathrm{K}$, et al. Prevalence of Type 2 (non-insulin-dependent) diabetes 
mellitus and impaired glucose tolerance in the Japanese general population: the Hisayama Study. Diabetologica. 1993;36:1198203.

41. Islam MM, Horibe H, Kobayashi F. Current trend in prevalence of diabetes mellitus in Japan, 1964-1992. J Epidemiol. 1999;9:15562.

42. Cui R, Iso H, Toyoshima H, Date C, Yamamoto A, Kikuchi S, et al. Body mass index and mortality from cardiovascular disease among Japanese men and women: the JACC study. Stroke. 2005;36:1377-82.

43. Yao M, Tachibana N, Okura M, Tanigawa T, Yamagishi K, Ikeda A, et al. Relationships of craniofacial morphology and body mass index with sleep-disordered breathing in Japanese men. Laryngoscope. 2004; $114: 1838-42$.

44. Noda H, Iso H, Toyoshima H, Date C, Yamamoto A, Kikuchi S, et al. Walking and sports participation and mortality from coronary heart disease and stroke. J Am Coll Cardiol. 2005;46:1761-7.

45. Tanigawa T. Obstructive sleep apnea: its prevention and screening may contribute to the prevention of hypertension, diabetes and cardiovascular diseases. EPMA J. 2011. doi:10.1007/s13167-0110073-2.

46. Matsumura Y. Nutrition trends in Japan. Asia Pac J Clin Nutr. 2001;10(Suppl):S40-7.

47. Shimamoto T, Komachi Y, Inada H, Doi M, Iso H, Sato S, et al. Trends for coronary heart disease and stroke and their risk factors in Japan. Circulation. 1989;79:503-15.

48. Yatsuya H, Yamagishi K, Iso H. Adiposity and risk of cardiovascular diseases in Japan: secular trend, individual level associations and causal pathway - implications for the prevention of cardiovascular diseases in societies with rapid economic development. EPMA J. 2011. doi:10.1007/s13167-011-0071-4.

49. Iso H, Kitamura A, Shimamoto T, Sankai T, Naito Y, Sato S, et al. Alcohol intake and the risk of cardiovascular disease in middleaged Japanese men. Stroke. 1995;26:767-73.

50. Ikehara S, Iso H, Toyoshima H, Date C, Yamamoto A, Kikuchi S, et al. Alcohol consumption and mortality from stroke and coronary heart disease among Japanese men and women: the Japan collaborative cohort study. Stroke. 2008;39:2936-42.

51. Kitamura A, Iso H, Sankai T, Naito Y, Sato S, Kiyama M, et al. Alcohol intake and premature coronary heart disease in urban Japanese men. Am J Epidemiol. 1998;147:59-65.

52. Kitamura A. Trends in alcohol intake among urban and rural Japanese populations. Nippon Koshu Eisei Zasshi. 1996;43:14252. in Japanese, English abstract.

53. Tanaka $\mathrm{H}$, Iso $\mathrm{H}$, Yokoyama $\mathrm{T}$, Yoshiike $\mathrm{N}$, Kokubo $\mathrm{Y}$. Cerebrovascular disease. In: Detels R, McEwen J, Beaglehole R, Tanaka H, editors. Oxford Textbook of Public Health, vol. 3. 4th ed. Oxford: Oxford University Press; 2002. p. 1193226.

54. Iso H, Shimamoto T, Naito Y, Sato S, Kitamura A, Iida M, et al. Effects of a long-term hypertension control program on stroke incidence and prevalence in a rural community in northeastern Japan. Stroke 1998;1510-8.

55. Noda H, Iso H, Saito I, Konishi M, Inoue M, Tsugane S, et al. The impact of the metabolic syndrome and its components on the incidence of ischemic heart disease and stroke: the Japan public health center-based study. Hypertens Res. 2009;32:289-98.
56. Irie F, Iso H, Noda H, Sairenchi T, Otaka E, Yamagishi K, et al. Associations between metabolic syndrome and mortality from cardiovascular disease in Japanese general population, findings on overweight and non-overweight individuals. Ibaraki Prefectural Health Study. Circ J. 2009;73:1635-42.

57. Committee to Evaluate Diagnostic Standards for Metabolic Syndrome. Definition and the diagnostic standard for metabolic syndrome. Nippon Naika Gakkai Zasshi. 2005;94:794-809. in Japanese.

58. Alberti KG, Zimmet P, Shaw J. Metabolic syndrome-a new world-wide definition. A Consensus Statement from the International Diabetes Federation. Diabet Med. 2006;23:469-80.

59. Kokubo Y, Okamura T, Yoshimasa Y, Miyamoto Y, Kawanishi K, Kotani Y, et al. Impact of metabolic syndrome components on the incidence of cardiovascular disease in a general urban Japanese population: the Suita study. Hypertens Res. 2008;31:2027-35.

60. Chei CL, Yamagishi K, Tanigawa T, Kitamura A, Imano H, Kiyama M, et al. Metabolic syndrome and the risk of ischemic heart disease and stroke among middle-aged Japanese. Hypertens Res. 2008;31:1887-94.

61. Saito I, Iso H, Kokubo Y, Inoue M, Tsugane S. Metabolic syndrome and all-cause and cardiovascular disease mortality: Japan Public Health Center-based Prospective (JPHC) Study. Circ J. 2009;73:878-84.

62. Yoon YS, Lee ES, Park C, Lee S, Oh SW. The new definition of metabolic syndrome by the international diabetes federation is less likely to indentify metabolically abnormal but non-obese individuals than the definition by the revised national cholesterol education program: the Korea NHANES Study. Int J Obes. 2007;31:528-34.

63. Gao W. Does the constellation of risk factors with and without abdominal adiposity associate with different cardiovascular mortality risk? Int J Obes (Lond). 2008;32:757-62.

64. Iso H. Changes in coronary heart disease risk among Japanese. Circulation. 2008;118:2725-9.

65. Okayama A, Ueshima H, Marmot MG, Nakamura M, Kita Y, Yamakawa M. Changes in total serum cholesterol and other risk factors for cardiovascular disease in Japan, 1980-1989. Int J Epidemiol. 1993;22:1038-47.

66. Kokubo Y, Iso H, Ishikawa J, Okada K, Inoue M, Tsugane S. The association of dietary intake of soy, beans and isoflavones with risk of cerebral and myocardial infarctions in the Japanese population: the Japan Public Health Center-Based (JPHC) Study Cohort I. Circulation. 2007;116:2553-62.

67. Naito Y. Relationship between physical activity and health examination variables in male workers - new methods to assess physical activity and their applications to epidemiologic research. Nippon Koshu Eisei Zasshi. 1994;41:706-19. in Japanese, English abstract.

68. Watanabe J, Iwabuchi K, Koseki Y, Fukuchi M, Shinozaki T, Miura M, et al. Declining trend in the in-hospital case-fatality rate from acute myocardial infarction in Miyagi Prefecture from 1980 to 1999. Jpn Circ J. 2001;65:941-6.

69. Konishi M, Komachi Y, Iso H, Iida M, Naito Y, Sato S, et al. Secular trends in atherosclerosis of coronary arteries and basal cerebral arteries in Japan. The Akita pathology. Arteriosclerosis. 1990;10:535-40.

70. Reddy KS, Yusuf S. Emerging epidemic of cardiovascular disease in developing countries. Circulation. 1998;97:596-601. 